\title{
MODELOS DE OCUPACIÓN PARA EL MONITOREO DE LA EFECTIVIDAD DE ESTRATEGIAS DE CONSERVACIÓN DEL ÁREA DE CONSERVACIÓN REGIONAL COMUNAL TAMSHIYACU TAHUAYO - ACRCTT, LORETO - PERÚ
}

\section{OCCUPANCY MODELS FOR THE MONITORING OF THE EFFECTIVENESS OF MANAGEMENT ACTIONS AT TAMSHIYACU TAHUAYO - ACRCTT, LORETO - PERÚ, COMMUNAL REGIONAL CONSERVATION AREA}

\author{
Emiliana Isasi-Catalá ${ }^{1}$, Julio Acosta ${ }^{2}$, Alejandra Anchante ${ }^{2}$, Guillermo Bianchi ${ }^{3}$, Jhonatan Caro ${ }^{2}$, Nereyda \\ Falconi $^{2}$, Leonardo Maffei ${ }^{2}$, Jorge Luis Martínez ${ }^{2}$ e Isaac Goldstein ${ }^{4}$
}

\begin{abstract}
Resumen
Evaluar la efectividad de acciones de manejo en áreas protegidas del Gran Paisaje Yavarí Samiria es esencial para la conservación y el uso sustentable de la biodiversidad de la región. El objetivo de este trabajo fue evaluar el uso de modelos de ocupación, como herramienta para monitorear la efectividad de los acuerdos comunales de extracción de especies. De febrero a mayo de 2015, se establecieron 84 cuadrículas de $1 \mathrm{~km}^{2}$ en el Área de Conservación Regional Comunal Tamshiyacu Tahuayo (ACRCTT), evaluando la ocupación de Tapirus terrestris (Linnaeus), Tayassu pecari (Link), Pecari tajacu (Linnaeus), Mazama sp., Cuniculus paca (Linnaeus), Dasyprocta sp., Dasypus sp. y Cracidae Vigors, a través de señales y rastros en transectos y estaciones de trampas-cámara. Se ajustaron modelos de ocupación single season-single species con covariables de sitio (CovS) para evaluar el efecto de la cacería. La ocupación ( $\psi$ ) estimada para ungulados fue alta $(\psi=0.95)$, excepto para $T$. pecari $(\psi=0.13)$, sin encontrar efecto de las covariables $(\operatorname{Sig} \beta(\mathrm{CovS})>0.05)$, indicando que la cacería no está afectando a estas especies en el ACRCTT. Para las especies pequeñas no fue posible ajustar modelos. La precisión de estas estimaciones permite su uso como indicadores confiables para el desarrollo de un programa de monitoreo de especies grandes en el ACRCTT.
\end{abstract}

Palabras clave: Acuerdos de manejo, áreas protegidas, cacería, especies cinegéticas, modelos de ocupación, monitoreo.

\begin{abstract}
Evaluate the effectiveness of management actions in protected areas of the Yavari Samiria Great Landscape is essential for the conservation and sustainable use of biodiversity in the region. The objective of this work was to assess the use of occupancy models as tools for monitoring the effectiveness of the sustainable use of some species by surrounding communities. Between February and May of 2015, at Tamshiyacu Tahuayo Communal Regional Conservation Area (ACRCTT), 84 grids of $1 \mathrm{~km}^{2}$, were established to evaluate the occupancy of Tapirus terrestris (Linnaeus), Tayassu pecari (Link), Pecari tajacu (Linnaeus), Mazama sp., Cuniculus paca (Linnaeus), Dasyprocta sp., Dasypus sp. and Cracidae vigors, through signals and trails in transects and camera traps stations. Single season-single species occupancy models with site covariates (CovS) were adjusted to assess the hunting effect. The occupancy estimated for ungulates was high ( $\Psi=0.95$ ), except for $T$. pecari $(\Psi=0.13$ ), with no effect of site covariates assessed $(\operatorname{Sig} \beta(\mathrm{CovS})>0.05)$. This indicates that hunting pressure is not affecting the large species evaluated at ACRCTT. It was not possible to adjust models for small species. The accuracy of these estimates indicates that the use of occupancy is a confident indicator for the development of a monitoring program of large species at ACRCTT.
\end{abstract}

Key words: Management settlements, protected areas, hunting, occupancy models, monitoring, hunted species.

\section{Introducción.}

En el Gran Paisaje Yavarí Samiria (Loreto, Perú), los sistemas naturales conformados por los bosques inundables, representan un área fundamental para la conservación y aprovechamiento de la biodiversidad de la Amazonía Andina peruana (ACRCTT, 2010). Allí, los programas de manejo deben estar enfocados no sólo a mantener los componentes de la 
biodiversidad sino a garantizar su aprovechamiento sustentable por parte de las comunidades locales (Bodmer et al., 1995; ACRCTT, 2010). El monitoreo, es la herramienta que permite evaluar los cambios en el estado de los recursos (Yoccoz et al., 2001), para de esta manera determinar las acciones de manejo que garanticen su aprovechamiento y conservación en el tiempo (Niemelä, 2000).

El ACRCTT fue establecida en el 2009, en respuesta a más de 25 años de solicitudes e iniciativas para el manejo sostenible por parte de las comunidades locales, enfocados en las especies que estaban siendo mermadas por la cacería descontrolada, como es el caso de los ungulados y grandes roedores (ACRCTT, 2010). El manejo de la cacería en el ACRCTT se basa en el establecimiento de acuerdos de extracción (ACRCTT, 2010), los cuales restringen las especies sujetas a explotación para uso y comercialización, el número de individuos por especie que puede extraerse, la frecuencia de extracción y la cantidad de munición disponible por cazador. Bajo estos acuerdos, se prohíbe la cacería de la sachavaca (Tapirus terrestris Linnaeus) y de los primates, como los del género Ateles E. Geoffroy y Cacajao calvus (I. Geoffroy). Para las especies grandes, se permite la cacería del sajino (Pecari tajacu Linnaeus) y la huangana (Tayassu pecari Link), limitada a cuatro individuos por cazador, de los cuales sólo se podrán comercializar tres. Mientras que para las especies pequeñas como el majaz (Cuniculus paca Linnaeus), añuje (Dasyprocta sp.) y armadillo (Dasypus sp.), se permite la cacería de cinco individuos, de los que se podrán comercializar cuatro. La frecuencia de cacería permitida es cada 60 días por cazador y los cazadores solo tienen 10 cartuchos por sesión de cacería. Otras especies no están incluidas en estos acuerdos, por lo que su cacería es libre, como es el caso del venado colorado (Mazama americana Erxleben), venado cenizo (Mazama nemorivaga F. Cuvier), pucacunga (Penelope jacquacu Spix) y paujil (Mitu tuberosum Spix) (ACRCTT, 2010). El objetivo de los acuerdos de extracción y manejo es garantizar la sostenibilidad de las poblaciones de especies animales sujetas a explotación, siendo fundamental evaluar si están siendo acatados, y si logran el objetivo de mantener las poblaciones en los niveles deseados.

Conocer el estado de las poblaciones animales, así como sus tendencias y amenazas, es la base del manejo y conservación de especies amenazadas (Gese, 2001). Los métodos clásicos, basados en densidad o índices de abundancia, suelen generar estimadores imprecisos, limitando el desarrollo de programas de monitoreo confiables (Guillerra-Arroita et al., 2010; Baker et al., 2011; Keane et al., 2012), para especies de bajas detectabilidades, como es el caso de especies de interés cinegético del ACRCTT (Pérez, 2012). Los modelos de ocupación pueden ser una alternativa eficiente para la evaluación de los cambios en el estado de las poblaciones de estas especies, ya que consideran la detectabilidad de la especie, generando estimadores exactos y precisos. (Royle \& Nichols 2003; MacKenzie et al., 2006). A partir de estos modelos jerárquicos, se puede estimar la probabilidad de que una especie se encuentre presente en un área o probabilidad de ocupación $(\Psi)$, así como su probabilidad de detección $(p)$, a partir de historiales de detección/no-detección (hi), obtenidos por el muestreo repetitivo ( $j$ visitas, donde $j=1,2, \ldots K$ ) de $S$ unidades o sitios de muestreo $i(i=1,2, \ldots S)$ (MacKenzie et al., 2002, 2006; Royle \& Nichols, 2003). Dependiendo de la escala espacial que se utilice, en relación al área de acción de la especie de interés, la probabilidad de ocupación puede interpretarse como distribución, ocupación, uso de hábitat y hasta abundancia (en el caso de especies territoriales), pudiendo modelarse la heterogeneidad entre sitios y/o visitas, a partir de covariables. Las características ambientales o la presencia de amenazas son las covariables más utilizadas en programas de monitoreo (Royle \& Nichols, 2003; MacKenzie et al., 2006). El objetivo de este trabajo fue evaluar los modelos de ocupación, como herramienta de monitoreo para determinar el estado de las poblaciones de especies cinegéticas y la efectividad de los acuerdos de manejo sobre el control de la cacería, bajo un enfoque de manejo adaptativo para esta reserva del Gran Paisaje Yavari-Samiria en Loreto, Perú.

\section{Materiales y métodos.}

Área de estudio.

El ACRCTT se encuentra dentro del Gran Paisaje Yavarí Samiria en la Región de Loreto (680 075 E - 9 528176 N y 768162 E, 9 444073, WGS 84, Figura. 1), contando actualmente con una superficie de 4200 $\mathrm{km}^{2}$. Presenta un clima húmedo y cálido, con una marcada estacionalidad. La temperatura promedio anual es de $26{ }^{\circ} \mathrm{C}$, llegando a máximas de $40{ }^{\circ} \mathrm{C}$ y mínimas de $14{ }^{\circ} \mathrm{C}$. La humedad relativa es de $85 \%$ y la precipitación total anual varía entre 2800 a 3200 mm (ACRCTT, 2010). En el área existe una importante red hidrográfica que sirve como único acceso a los pobladores, siendo los más importantes los ríos Tahuayo y Tamshiyacu, así como la quebrada Blanco. Los cauces de estos ríos presentan una dinámica importante tanto para la biodiversidad como para el uso de los recursos por parte de las comunidades, con una época de vaciante o estío de junio a octubre y una época de creciente de noviembre a mayo (ACRCTT, 2010). El ACRCTT mantiene ecosistemas amazónicos de altura, con una importante riqueza de especies, incluyendo especies amenazadas y de importancia cultural y económica para las comunidades locales (ACRCTT, 2010).

Diseño de muestreo.

Se establecieron al azar 84 cuadrículas de $1 \mathrm{~km}^{2}$ (84 km² en total), en el área de la cuenca de Quebrada 
Blanco (Figura 1), para el estudio de especies grandes (sachavaca, venados del género Mazama, sajino, huangana y primates grandes), y pequeñas (majaz, añuje, armadillos y crácidos). En estas se realizó la detección de las especies utilizando métodos no invasivos: búsqueda por avistamiento o señales y rastros en transectos y trampas-cámara. Para ello, se establecieron aleatoriamente tres transectos no lineales de aproximadamente $600 \mathrm{~m}$, ubicados desde los vértices al centro de la cuadrícula. En cada transecto se buscaron señales y rastros (huellas, heces, marcas, entre otros) o registros directos (avistamientos y cantos) que indiquen la presencia de las especies. Cada evidencia fue georreferenciada, registrada y organizada en una base de datos. Se establecieron estaciones de trampa-cámara (una por cuadrícula) en 50 de las 84 cuadrículas de muestreo, las cuales estuvieron operativas durante un máximo de 75 días. Las estaciones de trampas-cámara fueron colocadas en sitios donde las evidencias de presencia de las especies focales indiquen una alta probabilidad de detección de las mismas. La accesibilidad de las cuadrículas y vulnerabilidad ante presiones de cacería fue caracterizada con tres covariables cuantitativas relacionadas con la heterogeneidad espacial de cada celda: distancia lineal de las cuadrículas a la red hidrográfica que sirve de vías de acceso al área (D_RED), distancia real a los centros poblados (D_CP) y distancia lineal a los campamentos de cacería (D_CAMP). Estas covariables se calcularon a través del procesamiento y análisis de capas de información geográfica con Sistemas de Información Geográfico (SIG), utilizando ArcView 3.2®.

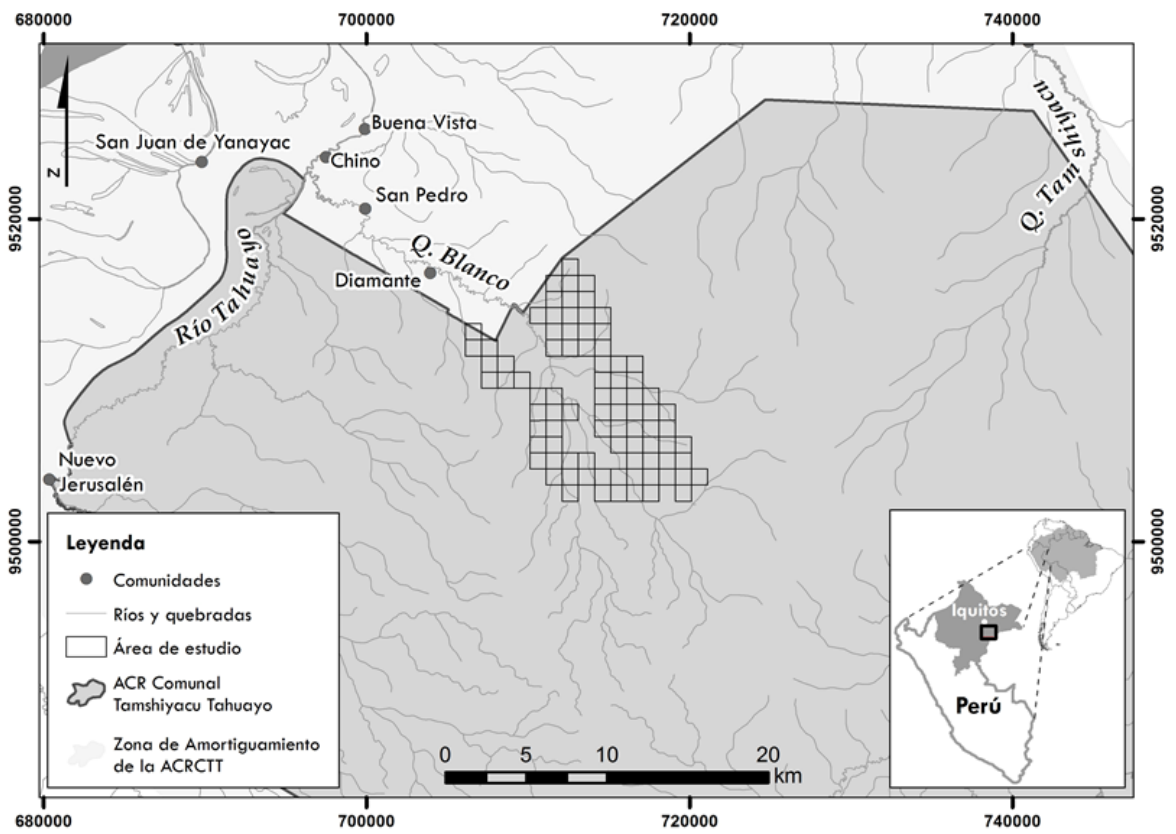

Figura 1. Área de Conservación Regional Comunal de Tamshiyacu Tahuayo (ACRCTT) y área de estudio utilizada durante el muestreo piloto del Programa de Monitoreo del ACRCTT. Elaborado por WCS-Perú.

\section{Análisis de datos}

Se construyeron tres matrices de historiales de detección/no-detección para las especies grandes, a partir de transectos $(\mathrm{T}, \mathrm{S}=84, \mathrm{~K}=3$ ) y trampascámara (TC, $S=50, K=5$ o 4, en función de la agrupación de los días de muestreo en bloques de 15 y 20 días continuos de muestreo): T, TC15, TC20. Adicionalmente se construyeron dos matrices combinadas para transectos y trampas-cámara $\mathrm{T}+$ TC15 ( $\mathrm{S}=50, \mathrm{~K}=8)$ y $\mathrm{T}+\mathrm{TC20}(\mathrm{S}=50, \mathrm{~K}=7)$. Con base en los modelos de ocupación single species single season (MacKenzie et al., 2002; 2006) se realizó la estimación de la probabilidad de ocupación ( $\psi$ ) y de la probabilidad de detección $(p)$ de cada especie, utilizando el programa Presence 9.7 (Hines, 2006). Se ajustaron modelos considerando covariables de sitio (tres covariables que pudieran afectar a $\psi$ : D_RED, D_CAMP y D_CP) para determinar los factores que afectan los patrones de ocupación de las especies (MacKenzie et al., 2002; 2006; Linkie, 2008). Sólo para los modelos basados en historiales de detección combinados, se empleó una covariable de muestreo (CvM) para evaluar el efecto del método sobre $p$ : CvM_MetodoMuestreo). Se evaluó el ajuste de cada modelo (bootstrap-1000 iteraciones: $\mathrm{pb}_{\mathrm{b}} \mathrm{y}$ sobredispersión de los valores: c), y se seleccionaron en función del delta del Criterio de Información de Akaike corregido para muestras pequeñas $(\triangle \mathrm{AICc})$. Se consideraron únicamente los modelos óptimos $(\triangle \mathrm{AICc}$ $\leq 3$ ) para la estimación de $\psi$ y $p$ (MacKenzie et al., 2006; Linkie, 2008). Se evaluó el efecto de las variables de sitio en su ocupación a partir de los coeficientes beta $(\beta)$, utilizando el test de Wald (Guillera-Arroita \& Lahoz-Monfort, 2012). Se calculó la potencia estadística obtenida a partir de este esfuerzo de muestreo y de las estimaciones de $\psi$ y $p$ utilizando el método descrito por GuilleraArroita \& LahozMonfort (2012). Esto con el objetivo de determinar si el indicador estimado es efectivo para desarrollar un programa de monitoreo que permita evaluar cambios poblacionales determinados, con un nivel de incertidumbre aceptable (e.g. detectar cambios en $\psi$ de estas especies de al menos $30 \%$, con una potencia 
$(1-\beta)$ de 0.80 y una significancia $(\alpha)$ de 0.20$)$.

\section{Resultados.}

Durante este estudio se establecieron 251 transectos, obteniéndose 1953 detecciones por señales y rastros de 23 especies: cuatro especies de aves y 19 especies de mamíferos. Las especies con mayor número de detecciones fueron el sajino, los venados (Mazama sp.), la sachavaca y el majaz, todas especies objeto de conservación del ACRCTT (Tabla 1). Con trampas-cámara se logró un esfuerzo de 3191 noches/trampa, con el que se obtuvieron 1346 detecciones de 19 especies. Las especies con más detecciones por trampas-cámara fueron el añuje, los venados, el majaz, el sajino y la sachavaca (Tabla 1).

Solo los modelos de ocupación obtenidos a partir de las detecciones por transecto presentaron ajustes adecuados para los ungulados (Tabla 1), por lo que son los que se utilizaron para el análisis del área ocupada por estas especies grandes (Tabla 1). Dado que no se encontraron efectos significativos de las covariables de sitio (CvS) sobre la ocupación de estas especies (incluso considerando $\mathrm{Sig} \beta(\mathrm{CvS})>0,10$, Tabla 1), los modelos finales utilizados para evaluar su ocupación fueron los nulos $(\triangle \mathrm{AICci} \leq 3)$, es decir, aquellos que no toman en cuenta covariables. Basados en los modelos nulos, la probabilidad de ocupación estimada para sachavaca fue de $\psi=0.97$ (EE 0.04), siendo la probabilidad de detección de $p=0.59$ (EE 0,04, Tabla 1). Para venados del genero Mazama, el escenario es similar, ya que los modelos nulos permitieron estimar una probabilidad de ocupación de $\psi=0.98$ (EE 0.03) y una probabilidad de detección de tan sólo $p=0.71$ (EE 0.03, Tabla 1). En el caso de los sajinos, los estimados de ocupación son de $\psi=0.98$ (EE 0.02), siendo la probabilidad de detección particularmente alta ( $p=0.80, \mathrm{EE}=0.03$, Tabla 1$)$. En el caso de las huanganas, las probabilidades de ocupación estimadas con el modelo nulo para esta especie resultaron muy bajas ( $\psi=0.13$, EE 0.05 ), y la probabilidad de detección baja e imprecisa $(p=0.41$, EE 0.12, Tabla 1). A partir de los historiales de detección combinados, se encontró un efecto significativo sobre la detectabilidad de sajino, sachavaca y venados del genero Mazama en función del tipo de muestreo realizado (Tabla 1). La detectabilidad de estas especies a partir de la búsqueda de señales y rastros por transectos resultó mayor ( $p_{T}$ mínimo $=0.63)$ que por trampas-cámara $\left(p_{T C}\right.$ mínimo $=0.26$ ).

Para el majaz, añuje, armadillo y los crácidos, sólo se pudieron generar historiales de detección a partir de las trampas-cámara, por lo que no fue posible ajustar modelos por transecto. A partir de trampas-cámara, los modelos obtenidos permiten estimar una ocupación mayor a 0.85 para majaz, añuje y armadillos, así como 0.50 para crácidos. Sin embargo, estos modelos presentaron problemas de ajuste y sobredispersión
(Tabla 1), por lo que las estimaciones de ocupación obtenidas no fueron confiables. En general los primates presentaron pocas detecciones (Tabla 1 ), por lo que no fue posible ajustar modelos ni siquiera al agrupar las especies según su tamaño: primates grandes (Alouatta sp., Callicebus cupreus Spix, Cebus sp. y Lagothrix lagotricha Humboldt) y pequeños (Saguinus sp. y Saimiri sciureus Linnaeus).

Las potencias estadísticas estimadas para sachavaca, sajino y venados del género Mazama, dado el esfuerzo de muestreo realizado y las estimaciones obtenidas, resultaron altas, para la evaluación de cambios del $20 \%$ y $30 \%$ de la ocupación de estos ungulados $(1-\beta)>0.80, \alpha=0.20$, Tabla 1). Para majaz y añuje, también se obtuvo estimaciones de potencia por encima de 0.80 (Tabla 1). Sin embargo, este resultado es limitado debido a las fallas en el ajuste de los modelos. Para el resto de las especies evaluadas (huangana, armadillo y crácidos), la potencia estadística resultó baja $(1-\beta<0.45, \alpha=$ 0.20 , Tabla 1 ).

\section{Discusión y recomendaciones finales.}

Modelos de ocupación como herramientas de monitoreo del ACRCTT

Para las sachavacas, venados del genero Mazama y sajinos, la potencia estadística estimada permite la detección de cambios de al menos un 20\% en la ocupación de estas especies, siendo estos modelos buenos indicadores para un programa de monitoreo del ACRCTT. Por tanto, utilizar el área ocupada por sachavaca, sajino y venados del género Mazama, estimada a partir de modelos de ocupación, es una buena alternativa para el establecimiento de un programa de monitoreo en el ACRCTT. Solo los modelos de ocupación obtenidos a partir de las detecciones por transecto presentaron ajustes adecuados (Tabla 1), razón por la que fueron utilizados para el análisis del área ocupada por sachavacas, venados del género Mazama y sajinos. Dados los resultados de los muestreos desarrollados solo con transectos y las implicaciones logísticas (al menos 2 visitas a las estaciones de fototrampeo) y los costos de los equipos de trampas-cámara, el diseño de muestreo de un programa de monitoreo en el ACRCTT para estos ungulados tiene que basarse en transectos. Tomando los resultados de este estudio piloto, un programa de monitoreo para evaluar la efectividad de los acuerdos de extracción establecidos en el ACRCTT pudiera estar basado en indicadores de ocupación estimados a partir de Modelos de Ocupación nulos single species - single season para sachavaca, sajino y Mazama sp. Para ello el diseño de muestreo podría basarse en el establecimiento de al menos 80 cuadrículas $(S=80)$ con tres visitas realizadas por transectos de $600 \mathrm{~m}(\mathrm{~K}=3)$, lo que permitiría la detección de cambios de menos del 30\% 
Tabla1: Área ocupada y probabilidad de detección de las especies evaluadas durante el estudio piloto del ACRCTT, utilizando covariables de sitio (CvS).

\begin{tabular}{|c|c|c|c|c|c|c|c|c|c|c|c|c|}
\hline \multirow{2}{*}{ Sp. } & \multirow{2}{*}{ Análisis } & \multirow{2}{*}{ Nd } & \multicolumn{3}{|c|}{ Ocupación } & \multicolumn{2}{|c|}{ Detección } & \multirow{2}{*}{ Ajuste } & \multirow{2}{*}{$\begin{array}{c}\alpha \\
\beta(\mathrm{CvS})\end{array}$} & \multirow{2}{*}{$\begin{array}{c}\alpha \\
\beta C v M)\end{array}$} & \multicolumn{2}{|c|}{$1-\beta$} \\
\hline & & & $\Psi_{\mathrm{N}}$ & $\Psi$ & ES & $\mathbf{p}$ & ES & & & & $\Delta 30 \%$ & $\Delta 20 \%$ \\
\hline \multirow{5}{*}{ T. terrestris } & TC15 & \multirow{2}{*}{136} & 0.54 & 0.60 & 0.08 & 0.48 & 0.05 & PA/PS & \multirow{2}{*}{\multicolumn{2}{|c|}{$\begin{array}{l}>0.05 \\
>0.05\end{array}$}} & - & - \\
\hline & TC20 & & 0.54 & 0.63 & 0.09 & 0.54 & 0.06 & $\mathrm{PA} / \mathrm{PS}$ & & & - & - \\
\hline & $\mathrm{T}$ & 301 & 0.90 & 0.97 & 0.04 & 0.59 & 0.04 & $\sqrt{ }$ & \multicolumn{2}{|l|}{$>0.05$} & 0.99 & 0.94 \\
\hline & $\mathrm{T}+\mathrm{TC} 15$ & & 0.96 & 0.97 & 0.03 & 0.45 & 0.03 & $\mathrm{PA} / \mathrm{PS}$ & $>0.05$ & 0.00 & - & - \\
\hline & $\mathrm{T}+\mathrm{TC} 20$ & & 0.96 & 0.98 & 0.03 & 0.50 & 0.03 & $\mathrm{PA} / \mathrm{PS}$ & $>0.05$ & 0.00 & - & - \\
\hline \multirow{5}{*}{ Mazama sp. } & TC15 & \multirow{2}{*}{283} & 0.84 & 0.90 & 0.05 & 0.59 & 0.04 & $\sqrt{ }$ & \multirow{2}{*}{\multicolumn{2}{|c|}{$\begin{array}{l}>0.05 \\
>0.05\end{array}$}} & - & - \\
\hline & TC20 & & 0.84 & 0.90 & 0.05 & 0.70 & 0.04 & $\sqrt{ }$ & & & - & - \\
\hline & $\mathrm{T}$ & 449 & 0.95 & 0.98 & 0.03 & 0.71 & 0.03 & $\sqrt{ }$ & \multicolumn{2}{|l|}{$>0.05$} & 0.99 & 0.99 \\
\hline & $\mathrm{T}+\mathrm{TC} 15$ & & 1.00 & 1.00 & 0.00 & 0.60 & 0.03 & $\sqrt{ }$ & $>0.05$ & 0.00 & - & - \\
\hline & $\mathrm{T}+\mathrm{TC} 20$ & & 1.00 & 1.00 & 0.00 & 0.67 & 0.03 & $\sqrt{ }$ & $>0.05$ & 0.01 & - & - \\
\hline \multirow{5}{*}{ T. pecari } & TC15 & \multirow{2}{*}{14} & 0.20 & 0.47 & 0.26 & 0.14 & 0.09 & PA/PS & \multirow{2}{*}{\multicolumn{2}{|c|}{$\begin{array}{l}>0.05 \\
>0.05\end{array}$}} & - & - \\
\hline & TC20 & & 0.20 & 0.30 & 0.12 & 0.33 & 0.14 & $\mathrm{PA} / \mathrm{PS}$ & & & - & - \\
\hline & $\mathrm{T}$ & 17 & 0.11 & 0.13 & 0.05 & 0.41 & 0.12 & $\sqrt{ }$ & \multicolumn{2}{|l|}{$>0.05$} & 0.41 & 0.31 \\
\hline & $\mathrm{T}+\mathrm{TC} 15$ & & 0.32 & 0.44 & 0.11 & 0.18 & 0.05 & $\mathrm{PA} / \mathrm{PS}$ & $>0.05$ & $>0.05$ & - & - \\
\hline & $\mathrm{T}+\mathrm{TC} 20$ & & 0.32 & 0.41 & 0.10 & 0.23 & 0.06 & $\mathrm{PA} / \mathrm{PS}$ & $>0.05$ & $>0.05$ & - & - \\
\hline \multirow{5}{*}{ P. tajacu } & TC15 & \multirow{2}{*}{141} & 0.86 & 1.00 & error & 0.45 & 0.04 & $\sqrt{ }$ & \multirow{2}{*}{\multicolumn{2}{|c|}{$\begin{array}{l}>0.05 \\
>0.05\end{array}$}} & - & - \\
\hline & TC20 & & 0.86 & 0.97 & 0.06 & 0.58 & 0.05 & $\sqrt{ }$ & & & - & - \\
\hline & $\mathrm{T}+$ & 517 & 0.98 & 0.98 & 0.02 & 0.80 & 0.03 & $\sqrt{ }$ & $>0.05$ & & 0.99 & 0.99 \\
\hline & $\mathrm{T}+\mathrm{TC} 15$ & & 1.00 & 1.00 & 0.00 & 0.61 & 0.03 & $\sqrt{ }$ & $>0.05$ & 0.00 & - & - \\
\hline & $\mathrm{T}+\mathrm{TC} 20$ & & 1.00 & 1.00 & 0.00 & 0.69 & 0.03 & $\sqrt{ }$ & $>0.05$ & 0.00 & - & - \\
\hline & TC15 & & 0.82 & 0.88 & 0.06 & 0.50 & 0.04 & $\mathrm{PA} / \mathrm{PS}$ & $>0.05$ & & 0.92 & 0.71 \\
\hline C. paca & TC20 & & 0.82 & 0.89 & 0.07 & 0.59 & 0.05 & $\mathrm{PA} / \mathrm{PS}$ & $>0.05$ & & 0.92 & 0.71 \\
\hline & $\mathrm{T}$ & 295 & & & & & de pos & le ajustar & stos moc & & & \\
\hline & TC15 & & 0.86 & 0.87 & 0.05 & 0.72 & 0.03 & $\mathrm{PA} / \mathrm{PS}$ & $>0.05$ & & 0.96 & 0.80 \\
\hline Dasyprocta sp. & TC20 & & 0.86 & 0.88 & 0.05 & 0.80 & 0.04 & $\mathrm{PA} / \mathrm{PS}$ & $>0.05$ & & 0.97 & 0.81 \\
\hline & $\mathrm{T}$ & 44 & & & & & de pos & le ajustar & stos moc & & & \\
\hline & TC15 & & 0.49 & 0.87 & 0.21 & 0.20 & 0.05 & PA/PS & $<0.05$ & & 0.39 & 0.28 \\
\hline Dasypus sp. & TC20 & & 0.49 & 0.99 & 0.27 & 0.23 & 0.07 & $\mathrm{PA} / \mathrm{PS}$ & $<0.05$ & & 0.35 & 0.27 \\
\hline & $\mathrm{T}$ & 175 & & & & & de pos & le ajustar & stos moc & & & \\
\hline & TC15 & & 0.28 & 0.55 & 0.22 & 0.18 & 0.07 & $\mathrm{PA} / \mathrm{PS}$ & $<0.05$ & & 0.29 & 0.24 \\
\hline Cracidae & TC20 & 3 & 0.27 & 0.50 & 0.19 & 0.24 & 0.10 & $\mathrm{PA} / \mathrm{PS}$ & $>0.05$ & & 0.28 & 0.23 \\
\hline & & 21 & & & & & le pos & le ajustar & stos moc & & & \\
\hline Primates & TC & 0 & & & & & de pos & le ajustar & stos moc & & & \\
\hline Grandes & $\mathrm{T}$ & 36 & & & & & le pos & le ajustar & stos moc & & & \\
\hline Primates & $\mathrm{TC}$ & 0 & & & & & 1e pos & e ajustar & stos moc & & & \\
\hline pequeños & $\mathrm{T}$ & 32 & & & & & de pos & le ajustar & stos moc & & & \\
\hline
\end{tabular}

T: transecto, TC15: trampa-cámara con ocasiones agrupadas cada 15 días, TC20: trampa-cámara con ocasiones agrupadas cada 20 días, Nd: número de detecciones, CvS: covariables de sitio, CvM: covariable de muestreo, $\Psi_{\mathrm{N}}$ : probabilidad de ocupación ingenua, $\Psi$ : probabilidad de ocupación estimada con el modelo, p: probabilidad de detección, $\alpha$ : significancia del efecto de beta de la covariable de sitio $(\mathrm{Ho}: \beta(\mathrm{CvS})=0)$ o muestreo $(\mathrm{Ho}: \beta(\mathrm{CvM})=0), 1$ - $\beta$ : potencia estadística alcanzada para lograr la detección de cambios de al menos $\Delta 30 \%$ y $\Delta 20 \%$ del área ocupada por la especie respectivamente (el valor mínimo de potencia aceptado es $80 \%$ ), EE. error estándar, PA: problemas diferencias entre los valores esperados y observados, PS: problemas de sobredispersión, $\sqrt{ }$ : modelos con ajuste adecuados. *Valores significativos.

del área ocupada para estas especies, con una potencia de 0.90 y una significancia de 0.10 .

En el ACRCTT, otras especies forman parte de los acuerdos de manejo, pero no pudieron ser evaluadas de manera adecuada en este estudio piloto. En el caso de huanganas, debido a que el ámbito de hogar de estas especie (home range, 21,8 a $200 \mathrm{~km}^{2}$, Keuroghlian et al., 2013) es mucho mayor al tamaño de la unidad de muestreo, los modelos obtenidos nos permiten evaluar el uso del hábitat de la especie y no de ocupación (MacKenzie et al., 2002; 2006; Royle \&
Nichols, 2003). Por otra parte, la potencia alcanzada a partir de los estimados de ocupación obtenidos no permite detectar el umbral de cambios establecido, por lo que sería necesario aumentar el esfuerzo de muestreo para alcanzar una potencia estadística que permita la detección de cambios de al menos un 30\%. Por tanto, establecer un muestreo para estimar la ocupación de huanganas implicaría unidades de muestreo de mayor tamaño y número, lo cual limita la utilización de la ocupación de esta especie como indicador dentro del programa de monitoreo de la 
ACRCTT. Especies como el majaz, añuje y armadillos, son blanco frecuente de cacería en la región, razón por la que fueron incluidas dentro del grupo de especies manejadas (ACRCTT, 2010; Pérez, 2012). Sin embargo, limitaciones de ajuste de los modelos por trampas-cámara hace que las estimaciones de ocupación obtenidas no sean confiables (Tabla 1), por lo que no fue posible evaluar la efectividad del estimador de ocupación como indicador del efecto de la presión de cacería sobre este grupo de especies manejadas del ACRCTT. A partir del muestreo por transectos, los modelos permitirían estimar la distribución de estas especies y no su ocupación, debido a las pequeñas áreas de ámbito de hogar de estas especies (Noon et al., 2012), lo que limita su implementación dentro de un programa de monitoreo. Por otra parte, tanto los primates como los crácidos son especies altamente sensibles a la cacería (Heymann \& Aquino, 1994; ACRCTT, 2010). Según los acuerdos de caza vigentes hasta el año 2015, los primates se encuentran entre las especies vedadas, mientras que los crácidos, pueden ser cazados libremente. Aun cuando hubo algunas detecciones de estas especies, el bajo número de registros no permitió el ajuste de modelos confiables, por lo que desconocemos su ocupación y la efectividad de indicadores basados en estos estimadores para la detección de cambios en un programa de monitoreo. $\mathrm{Si}$ se quiere incluir estas especies como parte del monitoreo en un futuro, se recomienda la implementación de un muestreo piloto enfocado en la detección tanto de especies terrestres pequeñas (añuje, majaz y armadillo) así como primates y crácidos, utilizando no sólo unidades de muestreo de tamaño adecuado según sus áreas de ámbito de hogar (Noon et al., 2012), sino métodos de campo adecuados para maximizar su detectabilidad (eg. detección por llamados o Callback en el caso de los primates grandes). De esta manera, se podría evaluar si estos cambios en el diseño de muestreo mejoran la detectabilidad de estas especies, obteniéndose estimadores de ocupación que permitan la detección de cambios con un grado de incertidumbre razonable. Ocupación de especies de interés cinegético en el ACRCTT.

Los estimados de ocupación obtenidos para sachavaca, venados del género Mazama y sajino, indican que actualmente estas especies ocupan toda el área de muestreo, por lo que se encuentran en buen estado de conservación en el área del estudio piloto de la reserva. Algunas de estas especies, como la sachavaca (NT) y el venado colorado (DD) están consideradas dentro de la lista de clasificación y categorización de las especies amenazadas de fauna silvestre legalmente protegidas del Perú, estando su cacería regulada o prohibida (MINARGI, 2014). En la cuenca del Tahuayo del ACRCTT, la cacería ha sido manejada por más de 15 años, a partir de los acuerdos que prohíben la cacería de sachavaca y limitan la de sajinos, mientras que permiten sin restricción la cacería de los venados (ACRCTT, 2010). El supuesto detrás de este marco de manejo es que las cuotas de extracción, acordadas con las comunidades, no afectan significativamente el estado de las poblaciones, haciendo de la cacería una práctica ecológicamente sostenible. El monitoreo de las poblaciones de estas especies, utilizando la ocupación como indicador, es la herramienta propuesta para evaluar si este supuesto es cierto o no. Bajo un escenario donde la presión de cacería en la zona del Tahuayo fuera importante para los ungulados, y los acuerdos de cacería se estuvieran cumpliendo, siendo la extracción acordada ecológicamente sostenible, se esperaba obtener estimados altos de ocupación para sachavaca y sajinos, más no así para los venados (cacería sin restricción). Por otra parte, bajo el mismo escenario de presión de caza y en el caso de que los acuerdos no se estuvieran cumpliendo, o si los niveles de extracción acordados no fueran ecológicamente sostenibles, se esperaba obtener los mismos resultados de ocupación para los tres tipos de especies, siendo la ocupación para todos los casos baja. La alta tasa de ocupación de sachavaca y sajino estimada (Tabla 1) pudiera indicar que los acuerdos de manejo y extracción suscritos por las cuatro comunidades más cercanas a la Quebrada Blanco se están cumpliendo, y los niveles de extracción acordados son ecológicamente sostenibles. Estos resultados pudieran indicar que estas medidas de manejo, establecidas e implementadas por las comunidades, pudieran ser la causa de la recuperación y mantenimiento de las poblaciones de estas especies, al regular la presión de cacería en la zona. Sin embargo, la ocupación de venados resultó igualmente alta, lo que indica que existe un escenario alternativo en el que la presión de cacería es naturalmente baja, sin importar los niveles de extracción propuestos o el cumplimiento o no de los acuerdos. Los resultados obtenidos indican que la actual presión de cacería realizada por los pobladores del Tahuayo en Quebrada Blanco no presenta un efecto significativo sobre la ocupación de estas especies de ungulados, independientemente del cumplimiento o no de los acuerdos. En este caso, la recuperación de las poblaciones de ungulados puede atribuirse a una disminución de la presión de cacería, debido a la diversificación de las actividades económicas desarrolladas por los pobladores (artesanía, pesca, agricultura) y la disminución de la densidad humana en la zona (ACRCTT, 2010). Las comunidades que actualmente aprovechan a estos ungulados, tienen pocos habitantes (Buenavista: 200 habitantes, El Chino: 330 habitantes, San Pedro: 140 habitantes y Diamante-7 de Julio: 120 habitantes), y se reporta la emigración de muchas familias buscando mejor calidad de vida y fuentes de trabajo (ACRCTT, 2010). Por tanto, el uso actual de estas especies para el 
sustento de las poblaciones del Tahuayo, no está generando un impacto significativo en las poblaciones de estos ungulados.

Dado el antecedente de sobre-cacería que llevó a las comunidades a establecer los acuerdos, mantener esta estrategia de manejo y monitorear el estado de las poblaciones aprovechadas es fundamental, ya que la situación de estas comunidades puede cambiar (las poblaciones locales pueden crecer o pueden venir cazadores de otras comunidades, y la cacería puede convertirse en la actividad económica principal de muchos pobladores). Por otra parte, desde inicio del año 2015, las comunidades establecieron un cambio en los acuerdos de caza, el cual implica un aumento de las cuotas de extracción (un animal adicional para especies grandes y pequeñas bajo manejo) y un cambio en las especies manejadas (los venados del género Mazama no podrán cazarse libremente sino bajo cuotas de extracción). Por tanto, monitorear el efecto de este cambio en la presión de cacería es una meta fundamental para asegurar la conservación y disponibilidad del recurso de caza para estas comunidades. La exactitud y precisión de los valores estimados obtenidos para sachavaca, sajino y Mazama sp., así como la alta potencia estadística alcanzada, garantizan no sólo la detección de cambios en la ocupación de estos ungulados en Quebrada Blanco, sino la posible evaluación de los factores que pudieran afectar en el futuro dicha ocupación, debido principalmente cambios en la presión de la cacería. Por ello, aun cuando actualmente no se observó un efecto de las covariables de sitio evaluadas, incluir covariables vinculadas con la accesibilidad y vulnerabilidad de cada sitio ante la cacería, es importante para evaluar posibles cambios en la ocupación de estas especies en el ACRCTT. Adicionalmente, los resultados obtenidos en este estudio reafirman la importancia de mantener, mejorar y reforzar el monitoreo de la presión de la cacería que realizan las comunidades del Tahuayo a partir de sus registros de caza, así como la necesidad de incluir el monitoreo del cumplimiento de los acuerdos establecidos por la comunidades. Este tipo de evaluación, no sólo serviría para apoyar la toma de decisiones de un proceso de manejo que garantice el mantenimiento de las poblaciones de estas especies legalmente protegidas y su aprovechamiento racional por parte de las comunidades locales, sino que serviría de instrumento para lograr la aprobación legal por parte de las instituciones del estado (MINAGRI, 2014), de las actividades de caza controlada que se llevan a cabo en el ACRCTT.

A partir de este estudio piloto se demuestra que los modelos de ocupación, son una alternativa adecuada para el monitoreo del estado de especies, como las especies objeto de conservación manejadas en el ACRCTT. Estos modelos no sólo permiten evaluar el estado de las poblaciones, sino que permiten entender el efecto directo de amenazas y otros factores ambientales que afectan la ocupación de las especies (Tobler et al., 2015). Por ello, los modelos de ocupación permiten evaluar tanto el estado de especies como sachavaca, venados del genero Mazama y sajinos, como la efectividad de los acuerdos manejo en el ACRCTT.

\section{Agradecimientos.}

Los autores agradecen a la Agencia de los Estados Unidos para el Desarrollo Internacional (USAID), que a través del Programa de Pequeñas Donaciones de la Iniciativa para la Conservación en la Amazonía Andina (ICAA) financió esta investigación. Agradecen a WCS, Programa Perú, por promover esta investigación. Agradecen a P. Huanca, D. Flores, L. Huanaquire, H. Huanaquiri, G. Caritimari, J. Gárate, A. Hidalgo, S. Cariajano, W. Vela y G. Velásquez por el levantamiento de información en campo. Agradecen al jefe del Parque Nacional del Manu, y al guardaparque G. Sonco por su apoyo y participación.

\section{Literatura citada.}

ACRCTT. 2010. Plan maestro del Área de Conservación Regional Comunal Tamshiyacu Tahuayo. Gobierno Regional de Loreto y Programa de Conservación, Gestión y Uso Sostenible de la Diversidad Biológica De Loreto - PROCREL.

Baker L., Arnold T., Olubode O. \& Garshelis D. 2011. Considerations for using occupancy surveys to monitor forest primates: a case study with Sclater's monkey (Cercopithecus sclateri). Population Ecology. 53: 549561.

Bodmer R., Penn J., Puertas P., Moya L. \& Fang T. 1995 Uniendo conservación y habitantes locales a través del uso sostenible de recursos naturales: Manejo Comunal en la Amazonía Peruana. Iquitos-Perú.

Gese E. 2001. Monitoring of terrestrial carnivore populations. En: J. Gittleman, S. Funk, D. Macdonald \& R. Wayne (editores) Carnivore conservation. Cambridge University Press, Cambridge, UK.

Guillera-Arroita G., Lahoz-Monfort J., Milner-Gulland E., Young R. \& Nicholson E. 2010. Using occupancy as a state variable for monitoring the Critically Endangered Alaotran gentle lemur, Hapalemur alaotrensis. Endangered Species Research. 11: 157-166.

Guillera-Arroita G. \& Lahoz-Monfort J. 2012. Designing studies to detect differences in species occupancy: power analysis under imperfect detection. Methods in Ecology and Evolution. 3: 860-869.

Heymann E. \& Aquino R. 1994. Exploraciones primatológicas en las quebradas Blanco, Blanquillo y Tangarana (río Tahuayo), Amazonia Peruana. Folia Amazonica. 6: 1-2.

Hines, J. E. (2006). PRESENCE- Software to estimate patch occupancy and related parameters. USGS-PWRC. http://www.mbr-pwrc.usgs.gov.html.

Keane A., Hobinjatovo T., Razafimanahaka H., Jenkins R. \& Jones J. 2012. The potential of occupancy modelling as a tool for monitoring wild primate populations: Monitoring wild primate populations using 
occupancy modelling. Animal Conservation. 15: 457465.

Keuroghlian A., Desbiez A., Reyna-Hurtado R., Altrichter M., Beck H., Taber A. \& Fragoso J.M.V. 2013. Tayassu pecari. The IUCN Red List of Threatened Species 2013: e.T41778A44051115. http://dx.doi.org/10.2305/IUCN.UK.20131.RLTS.T41778A44051115.en

Linkie M. 2008. Single-Species, Single-Season: Occupancy tutorials. DICE, University of Kent. UK.

Mackenzie D., Nichols J., Lachman G., Droege S., Royle J. \& Langtimm C. 2002. Estimating site occupancy rates when detection probabilities are less than one. Ecology. 83: 2248-2255.

Mackenzie D., Nichols J., Royle A., Pollock K., Bailey L. \& Hines J. 2006. Occupancy estimation and modeling. Inferring patterns and dynamics of species occurrence. Elsevier Academic Press. Oxford. UK.

MINAGRI - Ministerio de agricultura y riego del Perú, 2014. Decreto Supremo 004-2014-MINAGRI: Decreto Supremo que aprueba la actualización de la lista de clasificación y categorización de las especies amenazadas de fauna silvestre legalmente protegidas. Diario Oficial El Peruano, Año XXXI, N ${ }^{\circ}$ 12813, 520495.

Niemelä J. 2000. Biodiversity monitoring for decisionmaking. Ann. Zool. Fenici. 37: 307-317.

Noon B., Bailey T., Sisk K., \& McKelvey K. 2012. Efficient species-level monitoring at the landscape scale. Conservation Biology. 26:432-441.

Pérez P. 2012. Funcionalidad del Área de Conservación Regional Comunal Tamshiyacu Tahuayo: un análisis integral de la sostenibilidad de la caza. Reporte Técnico. WCS.

Royle J. \& Nichols J. 2003. Estimating abundance from repeated presence-absence data or point counts. Ecology. 84: 777-790.

Tobler M., Zúñiga Hartley A., Carrillo-Percastegui S. \& Powell, G. 2015. Spatiotemporal hierarchical modelling of species richness and occupancy using camera trap data. Journal of Applied Ecology. 52: 413-421.

Yoccoz N., Nichols J. \& Boulinier T. 2001 Monitoring of biological diversity in space and time. Trends Ecol. Evol. 16: 446-453.

${ }^{1}$ Fundación para la Defensa de la Naturaleza (FUDENA), Cortijos de Lourdes, 5-505 CE Senderos, AP-70776, Caracas 1071, Venezuela. Correo electrónico: zmiliana@gmail.com

${ }^{2}$ Wildlife Conservation Society Perú. Correos electrónicos: jjacosta@wcs.org; aanchante@wcs.org, jcttuesta@gmail.com; nereydafl@hotmail.com; lmaffei@wcs.org,jlmartinez@wcs.org

${ }^{3}$ Departamento de Medición y Evaluación. Faculta de Humanidades y Educación, Universidad de Los Andes. Av. Las Américas, Conjunto Universitario La Liria, Edificio A, Piso 2. Mérida 5101 Venezuela, Correo electrónico: gbianchi@ula.ve

${ }^{4}$ Wildlife Conservation Society, Global Conservation Program, 2300 Southern Boulevard, Bronx, New York, USA, Correo electrónico: igoldstein@wcs.org 